\title{
Tribulus terrestris $L$. protects glomerular endothelial cells via the miR155-H2AC6 interaction network in hypertensive renal injury
}

\author{
Hui-Juan Pei ${ }^{1 \#}$, Jie Yang ${ }^{2 \#}$, Fang-Xiao Hu ${ }^{1}$, Yong-Zhi Chen ${ }^{2}$, Chuan-Hua Yang ${ }^{2}$ \\ ${ }^{1}$ College of Traditional Chinese Medicine, Shandong University of Traditional Chinese Medicine, Jinan, China; ${ }^{2}$ Department of Cardiovascular, \\ Affiliated Hospital of Shandong University of Traditional Chinese Medicine, Jinan, China \\ Contributions: (I) Conception and design: H Pei, J Yang, C Yang; (II) Administrative support: J Yang, C Yang; (III) Provision of study materials or \\ patients: H Pei, J Yang; (IV) Collection and assembly of data: H Pei, F Hu, Y Chen, J Yang; (V) Data analysis and interpretation: F Hu, Y Chen; (VI) \\ Manuscript writing: All authors; (VII) Final approval of manuscript: All authors. \\ "These authors contributed equally to this work. \\ Correspondence to: Jie Yang; Chuan-Hua Yang. Department of Cardiovascular, Affiliated Hospital of Shandong University of Traditional Chinese \\ Medicine, Jinan, China. Email: sumuzheyangjie@163.com; yangchuanhua1962@126.com.
}

Background: Hypertensive renal injury is one of the most lethal complications of hypertension. At present, renin-angiotensin-aldosterone system (RAAS) blockers are considered the best drugs for the treatment of renal injury in hypertension because of their nephroprotective effect of reducing proteinuria, but there are no specific drugs for this purpose, however, clinical trials proved that Chinese medicine has a protective effect on target organs in the treatment of hypertension. Tribulus terrestris L. (TrT), a traditional Chinese medicine (TCM), has potential applications due to its reno-protective and immunomodulatory effects.

Methods: We investigated the underlying reno-protective mechanism of TrT on Angiotensin II (AngII)induced hypertensive renal injury in glomerular endothelial cells by integrating the differential expression profiles of micro RNA (miRNA) and messenger RNA (mRNA) to construct a miRNA-mRNA interaction network associated with hypertensive kidney injury, followed by quantitative real-time polymerase chain reaction (qRT-PCR) for validation.

Results: Seventy-six differentially expressed mRNAs (DEmRNAs) and 1 differentially expressed miRNAs (DEmiRNAs) were identified in the control group and the AngII-induced hypertensive renal injury group, respectively. 110 DEmRNAs and 27 DEmiRNAs were identified in the TrT treatment group and the AngIIinduced group, respectively. The core component of the miRNA-mRNA network was miR-155-5p. Our study showed that miR-155-5p expression levels were more decreased in the AngII-induced hypertensive renal injury group than the control group. TrT treatment also significantly upregulated miR-155-5p. Additionally, we found that miR-155-5p expression levels were negatively correlated with H2A clustered histone 6 (H2AC6).

Conclusions: The results of this study indicate that TrT has a reno-protective effect on AngII-induced hypertensive renal injury by miR-155-5p, which negatively regulates the expression of H2AC6. Our findings offer a new therapeutic strategy and have identified an effective candidate target for the treatment of hypertensive renal injury in clinical settings.

Keywords: Hypertension; kidney; Angiotensin II (AngII); micro RNA (miRNA)

Submitted Sep 29, 2021. Accepted for publication Nov 10, 2021.

doi: 10.21037/atm-21-5641

View this article at: https://dx.doi.org/10.21037/atm-21-5641 


\section{Introduction}

Hypertension is considered to be one of the major causes of chronic kidney disease (CKD). Hypertension is closely associated with renal disease and thought to damage the glomeruli of the kidney (1). With the advancement of research in recent years, it is believed that the pathological mechanism of renal damage caused by hypertension is mainly related to the renin-angiotensin-aldosterone system (RAAS), endothelial dysfunction, oxidative stress and inflammatory response, podocyte damage, calcium channel activation, hypoxia-inducible factor (HIF) overexpression, and genetic and epigenetic determinants $(2,3)$. The RAAS is one of the most important molecular mechanisms associated with hypertensive renal injury (4). Angiotensin II (AngII) can directly cause renal vascular smooth muscle cells to vasoconstrict (5), resulting in glomerular capillary injuries and the development of necrosis and fibrosis of the kidneys (3). The principal responses to renal injury include the inflammatory process, elevated glomerular protein filtration, and fibrosis leading to glomerular sclerosis $(3,6)$. Research has shown that the inflammatory effect of AngII plays a central role in the pathogenesis of hypertension and renal injury (1,7-10). Currently, among the drugs used to treat hypertension, only RAAS inhibitors have the effect of reducing proteinuria and slowing down the progression of CKD, but RAAS inhibitor monotherapy has significant benefits, and dual treatment with Angiotensin Converting Enzyme Inhibitor (ACEI) and Angiotensin II Receptor Blocker (ARB), instead of significant gains, can aggravate renal function damage (2). In contrast, a large number of clinical trials have shown that traditional Chinese medicine (TCM) has shown some advantages in protecting renal function in the treatment of hypertension.

Tribulus terrestris $L$. (TrT) is a plant that belongs to the Zygophyllaceae family. TrT is a TCM and is widely used in the treatment of cardiovascular disease, liver disease, and eye disease. In recent years, studies have shown that the pharmacological effects of TrT include immunomodulatory, antitumor, antioxidant, and antidiabetic effects $(11,12)$. Saponin, the main active ingredient of $\operatorname{Tr} T$, contributes to its pharmacological activities (13). To date, no study appears to have been conducted on the molecular mechanism by which TrT protects glomerular endothelial cells against AngII-induced renal injury.

Micro RNAs (miRNAs) are small (about 22 nucleotides in length) non-coding RNAs and post-transcriptional regulators of gene expression (14). miRNAs, produced by RNaseII-Dicer, precisely regulate the expression of the target genes by inhibiting the translation or guiding the cleavage of target messenger RNAs (mRNAs) (15). There is increasing evidence that many miRNAs are biomarkers and can be used as therapeutic targets in various human diseases $(16)$, such as renal injury $(17,18)$ and cardiovascular disease (19). MiR-155-5p has been shown to be significantly upregulated in salt-sensitive hypertensive kidney injury (20). MiR-192-5p is significantly lower in kidney biopsy specimens from patients with hypertension or hypertensive nephropathy compared with levels in controls (21). MiR191-5p has been shown to be downregulated in CKD patients with hypertension (22). Additionally, miR-103a$3 \mathrm{p}$ has been shown to be upregulated in hypertensive nephropathy patients, and miR-103a-3p/SNRK/NF-kB/ p65 is a regulatory axis of AngII-induced renal inflammation and fibrosis (1). These studies demonstrated that there was a strong association between miRNA expression levels and hypertensive renal injury; however, little is known about the miRNA regulation network that might be involved in AngII-induced hypertensive kidney injury.

In this paper, we performed an integrated analysis of miRNA and mRNA expression profiles to investigate the reno-protective effect of TrT in AngII-induced hypertensive kidney injury. Figure 1 shows the workflow of this study. First, the differentially expressed miRNAs (DEmiRNAs) and mRNAs (DEmRNAs) were identified. Next, the miRNAmRNA interaction network associated with hypertensive renal injury was identified. Finally, the miRNA-mRNA pairs were verified by quantitative real-time polymerase chain reaction (qRT-PCR). We found that the miR-155-5p expression level was more decreased in the AngII-induced hypertensive renal injury group than the control group. TrT treatment could significantly upregulate miR $-155-5 \mathrm{p}$. The miR-155-5p expression levels were negatively correlated with H2A clustered histone 6 (H2AC6). Taken together, our results indicate that $\operatorname{Tr} \mathrm{T}$ had a reno-protective effect on AngII-induced hypertensive renal injury in glomerular endothelial cells by miR-155-5p, which negatively regulated the expression of H2AC6. We present the following article in accordance with the MDAR checklist (available at https:// dx.doi.org/10.21037/atm-21-5641).

\section{Methods}

\section{Reagents}

The gross saponins of TrT were purchased from Shanghai Yuanye Bio-Technology Co., Ltd (B28061, Shanghai, China), and AngII was purchased from Beijing Solarbio 


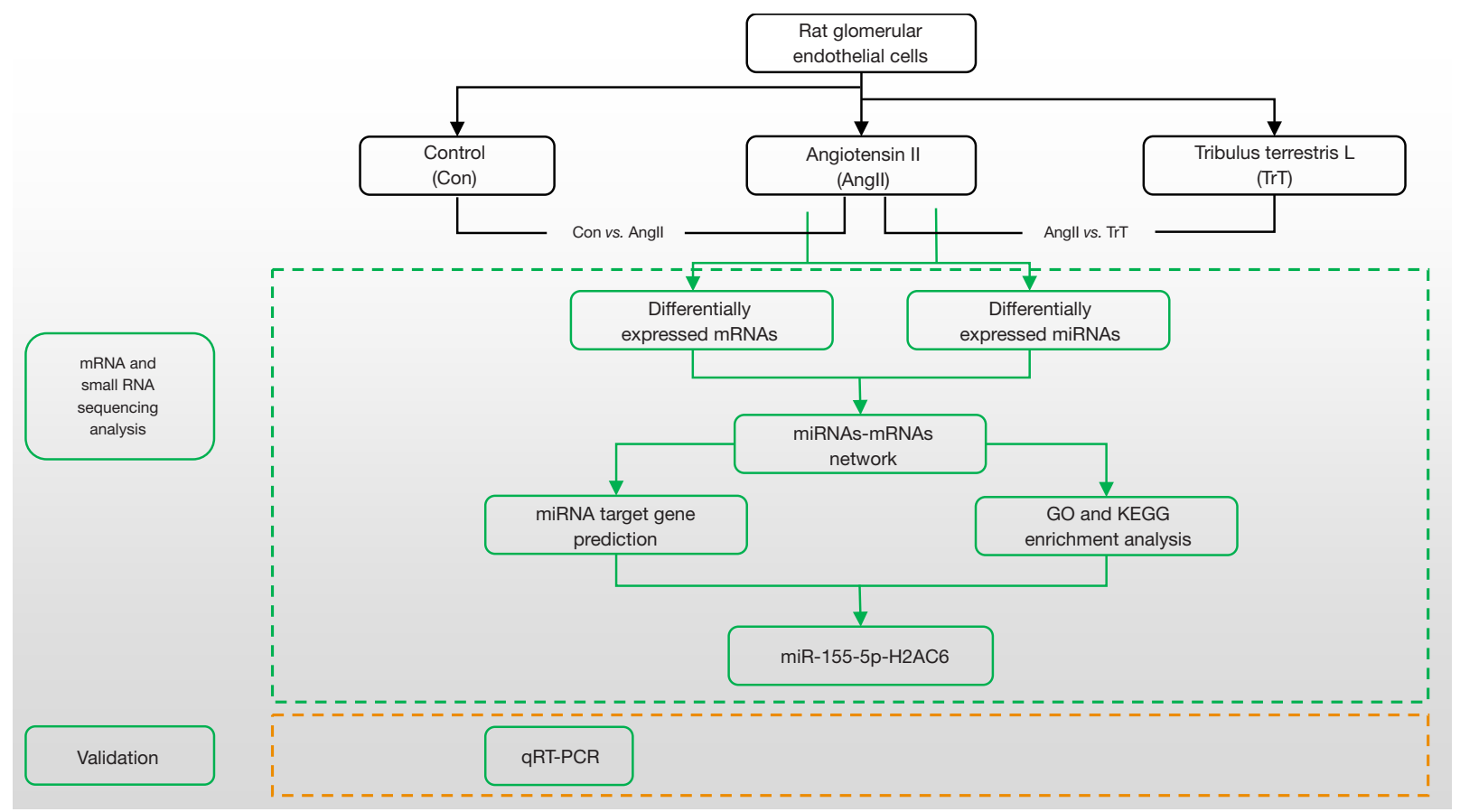

Figure 1 Overview of the data analysis workflow. There were 3 groups: the control group, the AngII group, and the TrT group treated with the gross saponins (saponins is the main active ingredient of TrT). First, we constructed the mRNA and miRNA libraries of each group, and sequenced the libraries on Illumina. Next, the DEmiRNAs and DEmRNAs in the AngII and the control groups, and the TrT treatment and AngII groups were analyzed. The miRNA-mRNA network was constructed using Pearson correlation coefficients. Based on the results of the Gene Ontology and Kyoto Encyclopedia of Genes and Genomes analyses and the predicted targets of miRNAs, we further validated the miRNA-mRNA pairs associated with hypertensive renal injury. mRNA, messenger RNA; miRNA, micro RNA; DEmiRNA, differentially expressed miRNA; DEmRNA, differentially expressed mRNA.

Science and Technology Co. Ltd (A9290, Beijing, China).

\section{$m R N A$ and small $R N A$ analysis}

\section{Cell culture and treatment}

WKY (Wistar-Kyoto) glomerular endothelial cells were obtained from Procell Life Science and Technology Co., Ltd (Wuhan, China) and cultured with Complete Medium for Rat Glomerular Endothelial Cells (Procell, China). The cells were randomized into the following 3 groups (each group had 3 replicates): (I) the control group (the cells were only cultured with medium); (II) the AngII group (the cells were induced with $100 \mu \mathrm{g} / \mathrm{mL}$ of AngII for $24 \mathrm{~h}$ ); and (III) the $\operatorname{Tr} T$ group (the cells were treated with $40 \mu \mathrm{g} / \mathrm{mL}$ of gross saponin from $\operatorname{Tr} T$ for $1 \mathrm{~h}$, and then induced with $100 \mu \mathrm{g} / \mathrm{mL}$ of AngII for $24 \mathrm{~h}$ ). To determine the appropriate concentration of TrT, we referred to Yuehua Jiang's experiment and set up three concentration gradients of 10 , 20 and $40 \mu \mathrm{g} / \mathrm{mL}$ (23), and the results showed that the high concentration group was suitable. Total RNA was isolated using isolater total RNA extraction reagent (Vazyme, China).

\section{RNA library preparation and Illumina sequencing RNA-Seq}

A total of 9 complementary DNA (cDNA) libraries were constructed for the 3 groups (i.e., the control group, the AngII group, and the TrT group). The cDNA libraries were prepared using the NEBNext ${ }^{\circledR}$ Ultra $^{\mathrm{TM}}$ RNA Library Prep Kit for Illumina ${ }^{\circledR}$ (NEB, USA) in accordance with the manufacturer's protocol. The library fragments of $370 \sim 420$ bp in length were purified with AMPure XP system (Beckman Coulter, Beverly, USA). The library preparations were then sequenced on the Illumina Novaseq platform, and 150 bp paired-end reads were generated.

\section{miRNA}

A total of 9 small RNA libraries were constructed for the 3 groups (i.e., the control group, the AngII group, and the $\operatorname{Tr} \mathrm{T}$ group). The small RNA libraries were prepared 
using the NEBNext ${ }^{\circledR}$ Multiplex Small RNA Library Prep Set for Illumina ${ }^{\circledR}$ (NEB, USA) in accordance with the manufacturer's protocol. The library preparations were then sequenced on Illumina Novaseq 6000 platform and $50 \mathrm{bp}$ single-end reads were generated.

\section{Differential expression analysis $R N A-S e q$}

The raw data were first processed to filter out any adapter and low-quality reads to obtain clean high-quality data. The clean data were aligned to the Rattus reference genome using HISAT2 v.2.0.5 (24). The gene expression levels were calculated by the expected number of Fragments Per Kilobase of transcript sequence per Millions base pairs sequenced (FPKM). The differentially expressed analysis was performed using the DESeq2 v.1.20.0 R package (25). The $\mathrm{P}$ values were adjusted using Benjamini and Hochberg's approach, and only genes with adjusted $\mathrm{P}$ values $<0.05$ were defined as differentially expressed DEmRNAs. The DEmRNAs of the 2 groups (i.e., the AngII vs. the control group, and the TrT treatment vs. the AngII group) were calculated.

\section{miRNA}

Clean data were mapped to the Rattus reference genome using Bowtie (26). The mapped reads were compared to miRBase22.0 using mirDeep2 to identify the known miRNA (27). The miRNA expression levels were estimated by transcript per million (TPM). The DEmiRNA in the AngII $v s$. the control group, and the TrT group $v s$. the AngII group were calculated using RNA-sequencing (RNA-Seq). Further, the target genes of the miRNAs were predicted by miRnada and RNAhybrid software $(28,29)$.

\section{Gene Ontology (GO) and Kyoto Encyclopedia of Genes and Genomes (KEGG) enrichment analysis}

The GO and KEGG enrichment analyses were performed by hypergeometric tests to identify significantly enriched GO terms and KEGG pathways in DEmRNAs and the predicted targets of miRNAs. Only the Go terms and KEGG pathways with $\mathrm{P}$ values $<0.05$ were considered significantly enriched.

\section{miRNA-mRNA interaction analysis}

A miRNA-mRNA interaction network was constructed to identify the key DEmiRNAs and DEmRNAs associated with renal lesion. The miRNA-mRNA interactions were calculated using Pearson correlation coefficients. Based on the miRNA-mRNA co-expression results, only mRNAs exhibiting negative relationships with miRNAs were used to construct the miRNA-mRNA interaction network $(30,31)$.

\section{qRT-PCR validation}

We further validated the key DEmiRNAs and DEmRNAs. WKY (Wistar-Kyoto) glomerular endothelial cells were cultured and treated under the same conditions described in Section "Cell culture and treatment", and were then randomized into the following 3 groups: (I) the control group (the cells were cultured normally without any other treatment); (II) the AngII group (the cells were induced with $100 \mu \mathrm{g} / \mathrm{mL}$ of AngII for $24 \mathrm{~h}$ ); (III) the $\operatorname{Tr} T$ group (the cells were treated with $40 \mu \mathrm{g} / \mathrm{mL}$ of gross saponin from $\operatorname{Tr} \mathrm{T}$ for $1 \mathrm{~h}$, and then induced with $100 \mu \mathrm{g} / \mathrm{mL}$ of AngII for $24 \mathrm{~h}$ ). The specific primers of miRNAs and mRNAs are listed in Table S1. The $2^{-\triangle \Delta C T}$ method was used to calculate the relative fold change of the house-keeping gene ( $\beta$-action).

\section{Statistical analysis}

All statistical analyses were performed using R 3.6.0. All the data are expressed as means \pm standard error. The 2 -tailed student $t$-test was used for data and the Wilcoxon ranksum test was used for abnormally distributed data. Data normality was evaluated using the Shapiro-Wilk test. The differences between 2 groups were considered significant at $\mathrm{P}$ value $<0.05$. $\mathrm{P}$ values were classified as * $\mathrm{P}<0.05$; **, $\mathrm{P}<0.01 ; * * *, \mathrm{P}<0.001$.

\section{Results}

\section{Identification of DEmRNAs and DEmiRNAs}

\section{mRNA}

For each group, 3 RNA libraries were sequenced. The mean raw read numbers of the RNA-seq libraries were 43972273, 47261794, and 44932087 for the control, AngII, and TrT treatment groups, respectively. Of the 76 DEmRNAs, 27 (35.53\%) upregulated and $49(64.47 \%)$ downregulated DEmRNAs were identified in the AngII vs. control group (see Figure 2A). Among these DEmRNAs, 6 (7.89\%) were new genes (see available online: https://cdn.amegroups.cn/ static/public/atm-21-5641-1.xlsx). Of the 110 DEmRNAs, $64(58.18 \%)$ upregulated and $46(41.82 \%)$ downregulated DEmRNAs were identified in the TrT treatment $v$ s. AngII group (see Figure 2B). Among these DEmRNAs, 11 (10\%) 

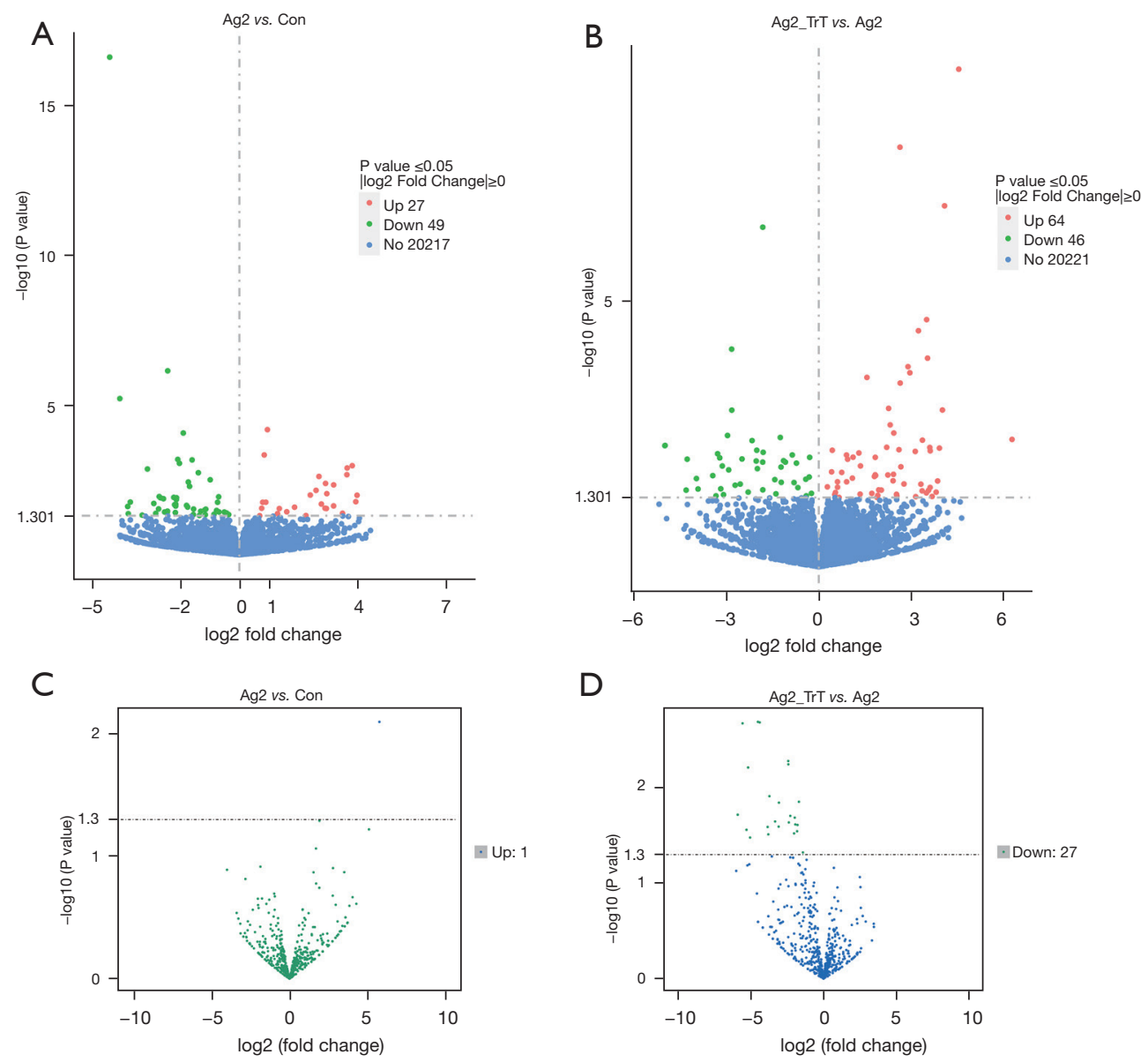

Figure 2 The DEmRNAs and DEmiRNAs in different comparisons. (A) Volcano plot of DEmRNAs in the AngII vs. control group; the red and blue dots represent upregulated and downregulated DEmRNAs. The $x$-axis shows the $\log 2$ (fold change), and the y-axis shows the $-\log 10$ (P value). (B) Volcano plot of DEmRNAs in the TrT treatment $v$ s. the AngII group. (C) Volcano plot of DE miRNAs in the AngII $v s$. the control group. (D) Volcano plot of DE miRNAs in the TrT treatment vs. the AngII group. Ag2, AngII group; Con, Control group; Ag2_TrT, TrT treatment group; mRNA, messenger RNA; miRNA, micro RNA; DEmiRNA, differentially expressed miRNA; DEmRNA, differentially expressed mRNA.

were new genes (see available online: https://cdn.amegroups. $\mathrm{cn} /$ static/public/atm-21-5641-2.xlsx). In addition, 21 same DEmRNAs were found in the two comparisons.

\section{miRNA}

For each group, 3 small RNA libraries were sequenced. The mean raw read numbers of the small RNA libraries were 11140012,26588885 , and 26588885 for the control, AngII, and TrT treatment groups, respectively. We analyzed DEmiRNAs using small RNA-seq data. In total, 1 upregulated and 27 downregulated DEmiRNAs were identified in the AngII $v$ s. the control group (see Figure 2C) and the TrT treatment vs. AngII group (see Figure 2D), respectively.

\section{GO and KEGG enrichment analysis}

We conducted a GO and KEGG enrichment analysis to analyze the functional role of DEmRNAs and the predicted targets of DEmiRNAs. In relation to the GO enrichment analysis, the following 3 categories of ontologies were identified: cellular component (CC), molecular function (MF), and biological process (BP).

\section{KEGG enrichment analysis of DEmRNAs}

The KEGG enrichment analysis of the AngII group and the control group (see Figure $3 A$ ) showed that the most 

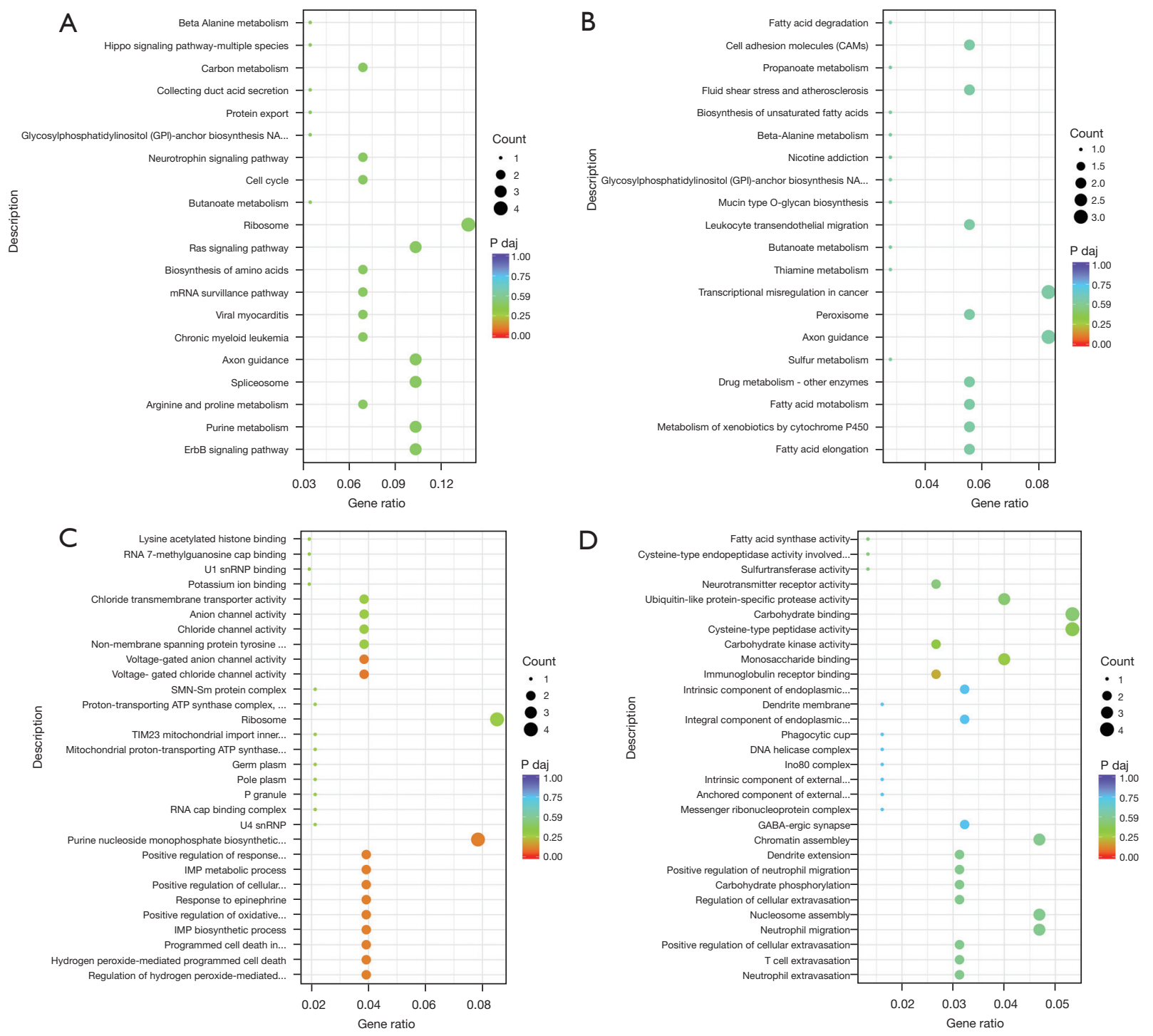

Figure 3 GO and KEGG enrichment analysis of DEmRNAs. (A) Top enriched KEGG pathways in the AngII vs. control group. (B) Top enriched KEGG pathways in the TrT treatment $v$ s. AngII group. (C) Top enriched GO terms in relation to the CC, MF, and BP in AngII vs. control group. (D) Top enriched GO terms in the TrT treatment vs. AngII group. Padj, p-adjusted (corrected P value). GO, Gene Ontology; KEGG, Kyoto Encyclopedia of Genes and Genomes; DEmRNA, differentially expressed mRNA; CC, cellular component; MF, molecular function; BP, biological process.

enriched pathway for DEmRNAs was the ErbB signaling pathway. The results showed that the metabolic pathways and viral myocarditis were also enriched. In relation to the KEGG enrichment analysis of the TrT treatment group and the AngII group (see Figure 3B), the DEmRNAs were principally enriched in the metabolic pathways, and involved in xenobiotic metabolism, fatty acid metabolism, drug metabolism, and sulfur metabolism. These pathways are associated with the pharmacological effects of TrT (32).

\section{GO analysis of DEmRNAs}

In relation to the AngII group and the control group, some BF terms (BP and MF) associated with proton channel activity, such as chloride channel activity, anion channel activity, and chloride transmembrane transporter activity, were highly enriched (see Figure 3C). The BP terms were principally enriched in hydrogen peroxide- 
mediated programmed cell death, the positive regulation of oxidative stress-induced cell death, and the regulation of hydrogen peroxide-induced cell death (see Figure 3C). In relation to the TrT treatment group and the AngII group, the terms were mainly related to immunoglobulin receptor binding, neutrophil extravasation, T-cell extravasation, and the positive regulation of neutrophil migration (see Figure 3D).

\section{DE miRNAs KEGG enrichment analysis}

In AngII vs. control group, the principally enriched pathways were metabolic-associated pathways, B cell receptor signaling pathways, and myeloid leukemia pathways (see Figure 4A). In the TrT treatment vs. AngII group, DEmiRNAs were mainly enriched in the Ras signaling pathway, axon guidance, and the metabolism pathways, such as the beta-alanine, fatty acid, and butanoate metabolism pathways (see Figure 4B).

\section{DE miRNAs GO enrichment analysis}

The most enriched CC term in the AngII vs. the control group was the NF-kappaB complex. The MF terms were principally enriched in the voltage-gated proton channel activities and immune systems, such as Immunoglobulin $\mathrm{G}$ receptor activity, and toll-like receptor 4 binding (see Figure 4C). In relation to the $\operatorname{Tr} T$ treatment $v$ s. the AngII group, the terms were associated with cell differentiation, cell development, ankyrin repeat binding, and the oxidationreduction process (see Figure 4D).

\section{miRNA-mRNA interaction analysis}

A total of 105 and 728 miRNA-mRNA pairs with negatively correlated expressions were identified in the AngII $v s$. control group, and the TrT treatment vs. AngII group, respectively. To identify the key molecular mechanism in hypertensive renal injury, we focused on the pairs associated with hypertensive renal injury based on the results of the GO and KEGG enrichment analyses. H2AC6 was targeted by 15 miRNAs (including 2 novel miRNAs), such as miR155-5p. H2AC6 was associated with the BP of chromatin silencing and the negative regulation of cell proliferation. Peroxisomal targeting signal 1 receptor ( PEX5) was targeted by 23 miRNAs (including 1 novel miRNA), such as miR-292-5p. PEX5 participated in the glucose metabolism and energy metabolism pathways. These results showed the key network of miRNA-mRNA interaction during hypertensive renal injury.

\section{qRT-PCR validation}

To verify the DEmRNAs and DEmiRNAs results, qRTPCR was used to confirm the miRNA-mRNA interaction networks (miR-155-5p- H2AC6). MiR-155-5p was more downregulated in the AngII group than the control group. $\operatorname{Tr} T$ treatment significantly increased the expression of miR-155-5p (see Figure 5). These results indicate that miR$155-5 \mathrm{p}$ may play a key role in hypertensive renal injury.

\section{Discussion}

Accumulated studies have proven that the pathogenesis of renal injury in hypertension is closely related to abnormalities in the renin-angiotensin system, increased activity of the sympathetic nervous system and salt load. Clinical studies by TCM scholars on renal injury in hypertension have shown that the use of TCM decoctions or their preparations in combination with western drugs is more advantageous in treating renal injury in hypertension, which can protect the kidneys while reducing blood pressure. Western antihypertensive drugs mainly reduce blood pressure to the ideal value to prevent and reduce renal injury, while TCM can significantly improve the symptoms of patients. For instance, Yan et al. showed that Jiang Ya Yi Shen granule (Jiangsu Province Hospital of TCM (Nanjing,

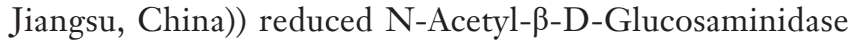
(NAG), albumin (Alb) and $\beta 2$-microglobin ( $\beta 2-M G)$ levels in the urethra of patients, decreased blood function parameters (including pulse index, mean velocity of the arterial blood, minimum velocity of the diastolic stage and peak velocity of the systolic wave) in the renal arteries, and significantly improved clinical symptoms in patients with hypertensive nephropathy, which was also verified in their animal studies, showing that this decoction may mediate microinflammation through inhibition of nuclear factor kappa $\beta$ signaling cytokines including interleukin-6 (IL-6), tumor necrosis factor- $\alpha$ (TNF- $\alpha$ ), intercellular cell adhesion molecule-1 (ICAM-1) and monocyte chemotactic protein 1 (MCP-1) exhibited protective effects in SHR (spontaneously hypertensive rats) (33).

In general, different TCM practitioners have adopted different Chinese medicine formulas to treat hypertensive kidney injury according to the principle of "syndrome differentiation and treatment" of TCM, which can lower the blood pressure and protect the kidney by inhibiting 

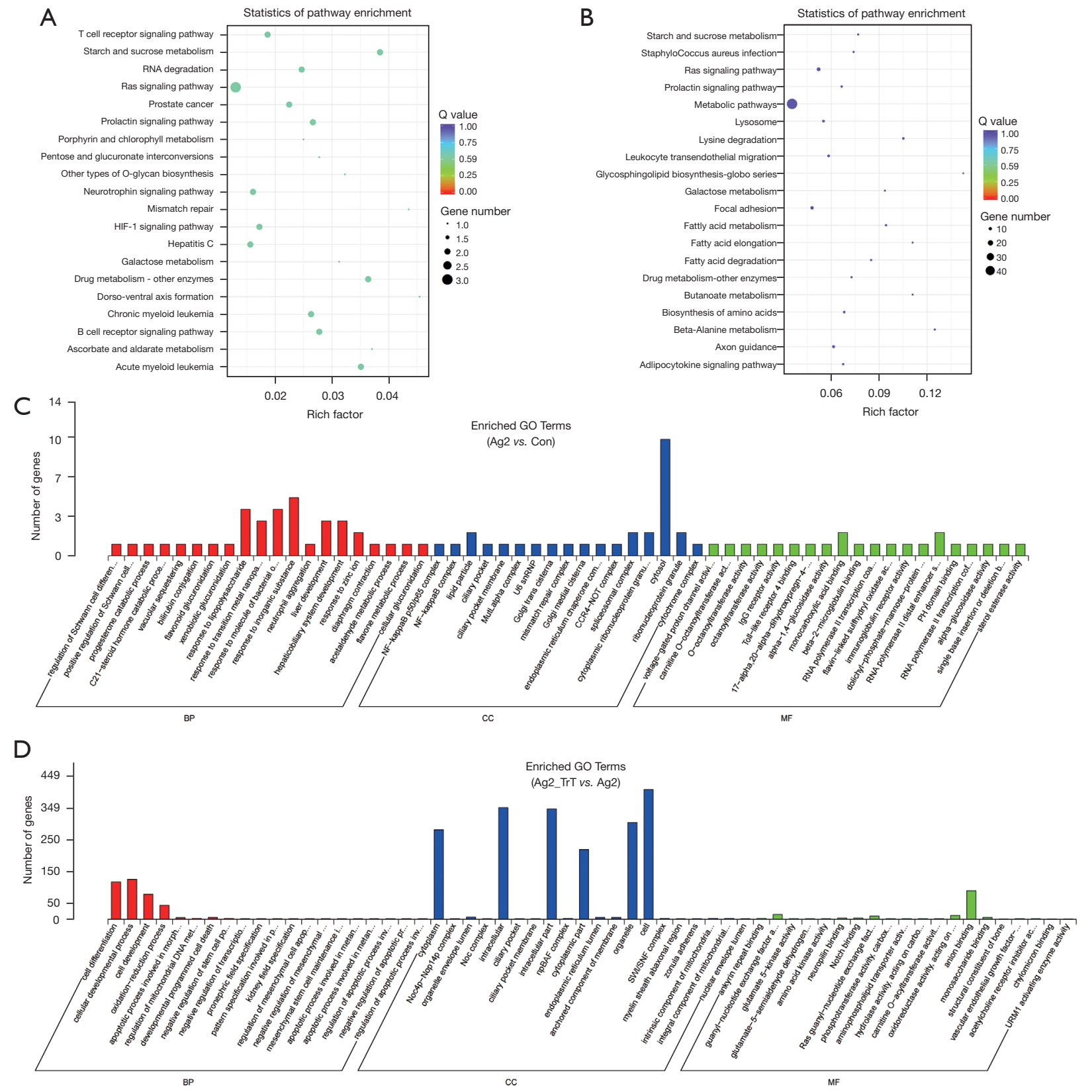

Figure 4 GO and KEGG enrichment analysis of DEmiRNAs. (A) Top enriched KEGG pathways of the AngII vs. control group. (B) Top enriched KEGG pathways of the TrT treatment vs. AngII group. (C) Top enriched GO terms in relation to the CC, MF, and BP in the AngII vs. control group. (D) Top enriched GO terms in the TrT treatment vs. AngII group. Ag2, AngII group; Con, Control group; Ag2 TrT, TrT treatment group; GO, Gene Ontology; KEGG, Kyoto Encyclopedia of Genes and Genomes; DEmiRNA, differentially expressed miRNA; BP, biological process; CC, cellular component; MF, molecular function.

the renal angiotensin system, reducing the excitability of sympathetic nervous system, anti-oxidation stress and antiinflammation, and regulating vasoactive substances (34). However, because the TCM decoctions are compound preparations, the current research cannot fully clarify their active ingredients and specific molecular mechanisms, and in-depth studies are still needed.

A data analysis study summarized 14 herbs (huangqi, 


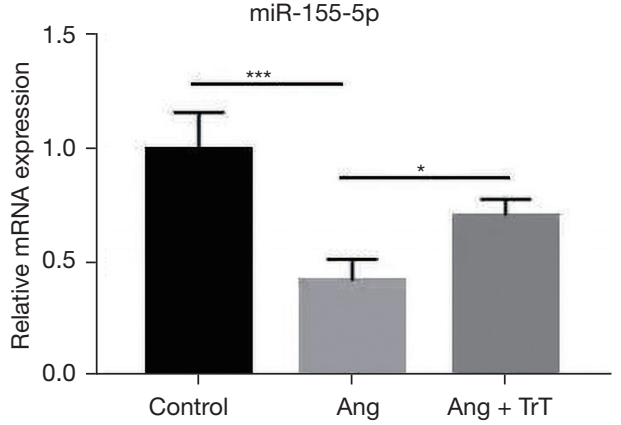

Figure 5 Validation of DEmiRNAs by qRT-PCR. *, $\mathrm{P}<0.05$; ***, $\mathrm{P}<0.001$. Control, Control group; Ang, AngII group; Ang + TrT, TrT treatment group.

baizhu, fuling, ezhu, cheqian zi, qumai, dangshen, danggui, danshen, chenpi, banxia, qianshi, niuxi and gancao) that are frequently used in the treatment of hypertensive nephropathy, and the analysis showed that they act through different biological pathways, such as reducing inflammatory cytokine expression through TNF, IL-1 $\beta$, IL- 6 and the NF$\kappa \mathrm{B}$ and MAPK signaling to reduce nephritis damage caused by RAAS overactivation. Moreover, these herbs can slow the decline of renal function and relieve the symptoms of hypertensive nephropathy (35).

In China, TrT is often used to treat hypertension, but the mechanism of its action in treating renal injury in hypertension is less studied. This appears to be the first study to systematically analyze miRNA and mRNA expression profiles to investigate the underlying molecular mechanism of $\operatorname{Tr} \mathrm{T}$ in protecting glomerular endothelial cells against AngII-mediated renal injury. TrT is a TCM that has been used for a long time in China in the treatment of various kinds of disease. The main biologically active ingredients of $\operatorname{Tr} T$ are saponins, flavonoids, and alkaloids. Saponins isolated from $\operatorname{Tr} \mathrm{T}$ have immunomodulatory, antidiabetic, hypolipidemic, and anticancer effects (36). Our results indicate that the gross saponin of $\operatorname{Tr} \mathrm{T}$ has a protective effect on hypertensive renal injury through miR$155-5 \mathrm{p}-\mathrm{H} 2 \mathrm{AC} 6$ interaction networks. This study extends understandings of the role of miR-155-5p in hypertensive renal injury, and suggests that miR-155-5p could be an effective candidate target for the treatment of hypertensive renal injury.

In this study, we performed an integrated analysis of miRNA and mRNA expression profiles in rat glomerular endothelial cells after AngII exposure. Seventy-six and 110 DEmRNAs were identified in the AngII vs. control group and the TrT treatment $v$ s. AngII group, respectively. One and $27 \mathrm{DE}$ miRNAs were identified in the AngII $v s$. control group and the TrT treatment vs. AngII group, respectively. The number of DEmRNAs and DEmiRNAs in the TrT treatment $v s$. AngII group was much greater than that of the AngII vs. control group.

Numerous studies have shown that miRNAs are biomarkers and therapeutic targets (37-40). Thus, we constructed a miRNA-mRNA interaction network to uncover the underlying molecular mechanism in hypertensive renal injury. Through the miRNA-mRNA network analysis, we found the miR-155-5p-H2AC6 regulatory axis may play a key role in hypertensive renal injury. H2AC6 is a core histone of nucleosome structures. H2AC6 has been shown to play key roles in heart failure, diabetic heart, atherosclerosis, and diabetic nephropathy (41-43). MiR-155-5p is expressed in various tissues and plays crucial roles in various pathological processes, including inflammation, immunity, tumor (44), cardiovascular disease, and kidney fibrosis processes (45-47). MiR-155 is a multifunctional miRNA with many target genes, which is involved in most life processes and plays an important role in related pathways (48). Therefore, based on the results of the present study, we speculate that miR-155-5p could regulate H2AC6 expression of AngIIinduced hypertensive renal injury in glomerular endothelial cells. MiR-155 has a bidirectional role in the inflammatory response and is closely related to inflammatory factors (48), while $\operatorname{Tr} \mathrm{T}$ can exert anti-inflammatory effects by altering the expression of inflammatory mediators and cytokines (49); therefore, TrT may resist hypertensive kidney injury by inhibiting the inflammatory response. It has been suggested that TrT may exhibit anti-hypertensive and endothelial protective effects through NF- $\mathrm{KB}$, and the transcription of miR-155 is regulated by NF- $\mathrm{KB}(48,50)$, so, we conjectured that one of the mechanisms of $\operatorname{Tr} T$ for treating hypertension kidney injury may be to regulate the expression of miR-155-5p through NF- $\mathrm{kB}$ pathway, which in turn regulates the expression of H2AC6, thus achieving renal protective effects.

In summary, this study proved that $\operatorname{Tr} \mathrm{T}$ has renoprotective effects on AngII-induced hypertensive renal injury in glomerular endothelial cells by integrating miRNA and mRNA expression profiles. TrT could upregulate miR-155-5p expression in glomerular endothelial cells under AngII exposure. MiR-155-5p expression levels were negatively correlated with H2AC6. Thus, TrT has a renoprotective effect on AngII-induced hypertensive renal injury 
by the miR-155-5p-H2AC6 network.

However, this study is also limited in that we only validated the expression of miR-155-5p, but not the expression of H2AC6 and the interaction between them. Another drawback is that we did not further investigate the specific molecular mechanism of renal injury in hypertension treated with $\operatorname{Tr} T$. In the future, we will conduct more in-depth research on this issue and expect to obtain satisfactory results.

\section{Acknowledgments}

Funding: This study was supported by the National Natural Science Foundation of China (No. 81804061), the 'Taishan Scholar' Project of Shandong Province (No. 201835), and the Ji'nan Science and Technology Project (No. 201805078). The funder had no role in study design, data collection and analysis, decision to publish, or preparation of the manuscript.

\section{Footnote}

Reporting Checklist: The authors have completed the MDAR checklist. Available at https://dx.doi.org/10.21037/ atm-21-5641

Data Sharing Statement: Available at https://dx.doi. org/10.21037/atm-21-5641

Conflicts of Interest: All authors have completed the ICMJE uniform disclosure form (available at https://dx.doi. org/10.21037/atm-21-5641). The authors have no conflicts of interest to declare.

Ethical Statement: The authors are accountable for all aspects of the work in ensuring that questions related to the accuracy or integrity of any part of the work are appropriately investigated and resolved.

Open Access Statement: This is an Open Access article distributed in accordance with the Creative Commons Attribution-NonCommercial-NoDerivs 4.0 International License (CC BY-NC-ND 4.0), which permits the noncommercial replication and distribution of the article with the strict proviso that no changes or edits are made and the original work is properly cited (including links to both the formal publication through the relevant DOI and the license). See: https://creativecommons.org/licenses/by-nc-nd/4.0/.

\section{References}

1. Lu Q, Ma Z, Ding Y, et al. Circulating miR-103a-3p contributes to angiotensin II-induced renal inflammation and fibrosis via a SNRK/NF- $\mathrm{kB} / \mathrm{p} 65$ regulatory axis. Nat Commun 2019;10:2145.

2. Sun D, Wang J, Shao W, et al. Pathogenesis and Damage Targets of Hypertensive Kidney Injury. J Transl Int Med 2020;8:205-9.

3. Mennuni S, Rubattu S, Pierelli G, et al. Hypertension and kidneys: unraveling complex molecular mechanisms underlying hypertensive renal damage. J Hum Hypertens 2014;28:74-9.

4. Li XC, Zhang J, Zhuo JL. The vasoprotective axes of the renin-angiotensin system: Physiological relevance and therapeutic implications in cardiovascular, hypertensive and kidney diseases. Pharmacol Res 2017;125:21-38.

5. Huang YS, Wang X, Feng Z, et al. Cordyceps cicadae Prevents Renal Tubular Epithelial Cell Apoptosis by Regulating the SIRT1/p53 Pathway in Hypertensive Renal Injury. Evid Based Complement Alternat Med 2020;2020:7202519.

6. Kurts C, Panzer U, Anders HJ, et al. The immune system and kidney disease: basic concepts and clinical implications. Nat Rev Immunol 2013;13:738-53.

7. Nair AR, Ebenezer PJ, Saini Y, et al. Angiotensin IIinduced hypertensive renal inflammation is mediated through HMGB1-TLR4 signaling in rat tubulo-epithelial cells. Exp Cell Res 2015;335:238-47.

8. Ding H, Zhou Y, Huang H. MiR-101a ameliorates AngIImediated hypertensive nephropathy by blockade of TGF $\beta /$ Smad3 and NF- $\kappa$ B signalling in a mouse model of hypertension. Clin Exp Pharmacol Physiol 2019;46:246-54.

9. Fähling M, Paliege A, Jönsson S, et al. NFAT5 regulates renal gene expression in response to angiotensin II through Annexin-A2-mediated posttranscriptional regulation in hypertensive rats. Am J Physiol Renal Physiol 2019;316:F101-12.

10. Foss JD, Fiege J, Shimizu Y, et al. Role of afferent and efferent renal nerves in the development of AngII-salt hypertension in rats. Physiol Rep 2018;6:e13602.

11. Zhu W, Du Y, Meng H, et al. A review of traditional pharmacological uses, phytochemistry, and pharmacological activities of Tribulus terrestris. Chem Cent J 2017;11:60.

12. Ștefănescu R, Tero-Vescan A, Negroiu A, et al. A Comprehensive Review of the Phytochemical, Pharmacological, and Toxicological Properties of Tribulus 
terrestris L. Biomolecules 2020;10:752.

13. Yuan Z, Du W, He X, et al. Tribulus terrestris Ameliorates Oxidative Stress-Induced ARPE-19 Cell Injury through the PI3K/Akt-Nrf2 Signaling Pathway. Oxid Med Cell Longev 2020;2020:7962393.

14. Sharma PC, Gupta A. MicroRNAs: potential biomarkers for diagnosis and prognosis of different cancers. Transl Cancer Res 2020;9:5798-818.

15. Li P, Gao Y, Li X, et al. mRNA and miRNA expression profile reveals the role of miR-31 overexpression in neural stem cell. Sci Rep 2020;10:17537.

16. Rupaimoole R, Slack FJ. MicroRNA therapeutics: towards a new era for the management of cancer and other diseases. Nat Rev Drug Discov 2017;16:203-22.

17. Brandenburger T, Salgado Somoza A, Devaux Y, et al. Noncoding RNAs in acute kidney injury. Kidney Int 2018;94:870-81.

18. van Zonneveld AJ, Rabelink TJ, Bijkerk R. miRNACoordinated Networks as Promising Therapeutic Targets for Acute Kidney Injury. Am J Pathol 2017;187:20-4.

19. Barwari T, Joshi A, Mayr M. MicroRNAs in Cardiovascular Disease. J Am Coll Cardiol 2016;68:2577-84.

20. Lu C, Chen B, Chen C, et al. CircNr1h4 regulates the pathological process of renal injury in salt-sensitive hypertensive mice by targeting miR-155-5p. J Cell Mol Med 2020;24:1700-12.

21. Baker MA, Wang F, Liu Y, et al. MiR-192-5p in the Kidney Protects Against the Development of Hypertension. Hypertension 2019;73:399-406.

22. Berillo O, Huo KG, Fraulob-Aquino JC, et al. Circulating let-7g-5p and miR-191-5p Are Independent Predictors of Chronic Kidney Disease in Hypertensive Patients. Am J Hypertens 2020;33:505-13.

23. Jiang YH, Jiang LY, Wu S, et al. Proteomic Analysis Reveals the Renoprotective Effect of Tribulus terrestris against Obesity-Related Glomerulopathy in Rats. Biol Pharm Bull 2018;41:1430-9.

24. Mortazavi A, Williams BA, McCue K, et al. Mapping and quantifying mammalian transcriptomes by RNA-Seq. Nat Methods 2008;5:621-8.

25. Love MI, Huber W, Anders S. Moderated estimation of fold change and dispersion for RNA-seq data with DESeq2. Genome Biol 2014;15:550.

26. Langmead B, Trapnell C, Pop M, et al. Ultrafast and memory-efficient alignment of short DNA sequences to the human genome. Genome Biol 2009;10:R25.

27. Friedländer MR, Mackowiak SD, Li N, et al. miRDeep2 accurately identifies known and hundreds of novel
microRNA genes in seven animal clades. Nucleic Acids Res 2012;40:37-52.

28. Enright AJ, John B, Gaul U, et al. MicroRNA targets in Drosophila. Genome Biol 2003;5:R1.

29. Krüger J, Rehmsmeier M. RNAhybrid: microRNA target prediction easy, fast and flexible. Nucleic Acids Res 2006;34:W451-4.

30. Kanehisa M, Araki M, Goto S, et al. KEGG for linking genomes to life and the environment. Nucleic Acids Res 2008;36:D480-4.

31. Young MD, Wakefield MJ, Smyth GK, et al. Gene ontology analysis for RNA-seq: accounting for selection bias. Genome Biol 2010;11:R14.

32. Guo $W$, Wang $Y$, Fan $M$, et al. Integrating metabolomics and network pharmacology to explore the protective effect of gross saponins of Tribulus terrestris L. fruit against ischemic stroke in rat. J Ethnopharmacol 2020;263:113202.

33. Yan D, Yue B, Qian M, et al. JYYS Granule Mitigates Renal Injury in Clinic and in Spontaneously Hypertensive Rats by Inhibiting NF- $\kappa$ B Signaling-Mediated Microinflammation. Evid Based Complement Alternat Med 2018;2018:8472963.

34. Dong Z, Dai H, Feng Z, et al. Mechanism of herbal medicine on hypertensive nephropathy (Review). Mol Med Rep 2021;23:234.

35. Huan JM, Su WG, Li W, et al. Summarizing the Effective Herbs for the Treatment of Hypertensive Nephropathy by Complex Network and Machine Learning. Evid Based Complement Alternat Med 2021;2021:5590743.

36. Chhatre S, Nesari T, Somani G, et al. Phytopharmacological overview of Tribulus terrestris. Pharmacogn Rev 2014;8:45-51.

37. Liu R, Yang L, Wei Q. miR-34a targets PAI-1 to regulate urinary microalbumin and renal function in hypertensive mice. Eur J Med Res 2020;25:3.

38. Chen C, Lu C, Qian Y, et al. Urinary miR-21 as a potential biomarker of hypertensive kidney injury and fibrosis. Sci Rep 2017;7:17737.

39. Wang G, Wu L, Chen Z, et al. Identification of crucial miRNAs and the targets in renal cortex of hypertensive patients by expression profiles. Ren Fail 2017;39:92-9.

40. Chen Z, Wu C, Liu Y, et al. ELABELA attenuates deoxycorticosterone acetate/salt-induced hypertension and renal injury by inhibition of NADPH oxidase/ ROS/NLRP3 inflammasome pathway. Cell Death Dis 2020;11:698.

41. Gao C, Chen G, Liu L, et al. Impact of high glucose and proteasome inhibitor MG132 on histone H2A and H2B 
ubiquitination in rat glomerular mesangial cells. J Diabetes Res 2013;2013:589474.

42. Yerra VG, Advani A. Histones and heart failure in diabetes. Cell Mol Life Sci 2018;75:3193-213.

43. Jiang W, Agrawal DK, Boosani CS. Cell-specific histone modifications in atherosclerosis (Review). Mol Med Rep 2018;18:1215-24.

44. Li N, Cui T, Guo W, et al. MiR-155-5p accelerates the metastasis of cervical cancer cell via targeting TP53INP1. Onco Targets Ther 2019;12:3181-96.

45. Bai X, Luo Q, Tan K, et al. Diagnostic value of VDBP and miR-155-5p in diabetic nephropathy and the correlation with urinary microalbumin. Exp Ther Med 2020;20:86.

46. Jiang K, Hu J, Luo G, et al. miR-155-5p Promotes Oxalate- and Calcium-Induced Kidney Oxidative Stress Injury by Suppressing MGP Expression. Oxid Med Cell

Cite this article as: Pei HJ, Yang J, Hu FX, Chen YZ, Yang CH. Tribulus terrestris $L$. protects glomerular endothelial cells via the miR155-H2AC6 interaction network in hypertensive renal injury. Ann Transl Med 2021;9(21):1626. doi: 10.21037/atm-215641
Longev 2020;2020:5863617.

47. Zhang W, Li X, Tang Y, et al. miR-155-5p Implicates in the Pathogenesis of Renal Fibrosis via Targeting SOCS1 and SOCS6. Oxid Med Cell Longev 2020;2020:6263921.

48. Li CC, Zhao JY, Wu J, et al. Research Progress on miR155. Biotechnology Bulletin 2018;34:70-82.

49. Lee HH, Ahn EK, Hong SS, et al. Anti-inflammatory effect of tribulusamide D isolated from Tribulus terrestris in lipopolysaccharide-stimulated RAW264.7 macrophages. Mol Med Rep 2017;16:4421-8.

50. Jiang YH, Guo JH, Wu S, et al. Vascular protective effects of aqueous extracts of Tribulus terrestris on hypertensive endothelial injury. Chin J Nat Med 2017;15:606-14.

(English Language Editor: L. Huleatt) 
Supplementary

Table S1 qRT-PCR primer sequences

\begin{tabular}{lll}
\hline miRNA or mRNA & Forward primer (5'-3') & Reverse primer (5'-3') \\
\hline miR-155-5p & GCGCGTTAATGCTAATTGTGAT & AGTGCAGGGTVVGAGGTATT \\
GAPDH & GCACCGTCAAGGCTGAGAAC & TGGTGAAGACGCCAGTGGA \\
$\beta$-actin & AAGGAGCCCCACGAGAAAAAT & ACCGAACTTGCATTGATTCCAG
\end{tabular}

GAPDH, Glyceraldehyde-3-Phosphate Dehydrogenase. 